\title{
Assessment of $\mathrm{AMBR}^{\mathrm{TM}}$ as a model for high-throughput cell culture process development strategy
}

\author{
Sarath Moses ${ }^{1 *}$, Matthew Manahan ${ }^{1}$, Alexandre Ambrogelly ${ }^{2}$, Wai Lam W. Ling ${ }^{1^{*}}$ \\ ${ }^{1}$ Upstream Process Development \& Operations, Bioprocess Development, Merck Research Laboratories, Union, USA \\ ${ }^{2}$ Analytical Development, Bioprocess Development, Merck Research Laboratories, Union, USA \\ Email: ${ }^{*}$ sarath.moses@merck.com, ${ }^{*}$ wai_lam.ling@merck.com
}

Received 20 August 2012; revised 27 September 2012; accepted 11 October 2012

\begin{abstract}
The development and delivery of high quality therapeutic products necessitates the need for highthroughput (HTP) process development tools. Traditionally, these works requires a combination of shake flask and small-scale stirred tank bioreactor (STR) which are labor and resource intensive and time-consuming. Here we demonstrate a strategy for rapid and robust cell culture process development by evaluating and implementing the use of a new HTP disposable micro bioreactor (MBR) called AMBR ${ }^{\mathrm{TM}}$ system (Advanced Microscale Bioreactor) that has the capabilities for automated sampling, feed addition, $\mathrm{pH}$, dissolved oxygen (DO), gassing and agitation controls. In these studies the performance of two monoclonal antibody (MAb) producing cell lines (MAb1 and MAb2) was evaluated both in the AMBR system and 3-L STR. We demonstrated that cell culture performance (growth and viability, production titer and product quality) were similar in both vessel systems. Furthermore, process control and feed optimization were demonstrated in an additional cell line (MAb3) in the disposable MBR and its performance confirmed at STR scale. The results indicate that the AMBR system can be used to streamline the process development effort and facilitate a rapid and robust cell culture process development effort for MAb programs in a HTP manner.
\end{abstract}

Keywords: Microbioreactors; High-Throughput; DoE; Process Ranging; Process Development; Cell Culture

\section{INTRODUCTION}

The continuing trend in biopharmaceutical industry in delivering quality therapeutic products into the market with shrinking timelines and limited resources has facilitated the development and implementation of HTP sys-

${ }^{*}$ Corresponding authors. tems to enhance data outputs and program decision in the cell culture process development area. In addition, recent ICH (International Conference on Harmonisation of Technical Requirements for Registration of Pharmaceuticals for Human Use) endorsed guidelines for ICH Q8/Q9/ Q10/Q11 implementation, [1] focuses on ensuring the quality of the therapeutic products to be safe and efficacious, and provides guidance on risk assessment and management on product quality control through identifycation of critical quality attributes (CQAs) and critical process parameters (CPPs). Our ongoing efforts in applying Quality by Design (QbD) approaches in high throughput cell culture systems are one of the strategies in assessing the product quality changes during development while balancing the resource and timeline constraints.

The ongoing efforts in the biopharmaceutical industry to balance the increased workload required to address process and product quality control coupled with the reduced resources availability have heightened the implementation of HTP systems to support development work. One of the HTP strategies utilized in upstream process development is the use of disposable microbioreactors for cell culture process development These disposable micro-bioreactors are expected to perform in a high-throughput manner under controlled bioreactor operation conditions similar to those at bench and largescale, but without extensive bioreactor set-up and cleanup procedures. These systems may replace the use of shake flasks for early stage of cell culture process development and optimization studies [2-5].

Recent literature have demonstrated availability of various designs of micro-scale reactors from a simpler standard plate with integrated sensors called Microtiter plate based bioreactors (MTB) [6-12] to more advanced cell culture systems such as the Stirred Mini Bioreactors (MBR) with control capabilities close to bench-top bioreactors for high throughput cell culture screening and optimization studies [13-17]. MBRs possess the advantage of being able to closely mirror the bench-scale bio- 
reactors in their monitoring and control capabilities and hence are considered as a preferred alternative vessel over shake flasks or MTBs for early stage process development. MBRs are generally equipped with optical probes to monitor $\mathrm{pH}$ and DO online and it has been extensively characterized for its oxygen transfer rate, mixing capabilities and gassing efficiency [13-17]. The above mentioned characteristics of MBRs typically make them an ideal set up for early high-throughput clonal screening efforts as part of process development.

In order to assess our HTP cell culture process development needs the ideal system must address the following requirements: 1) be able to individually control $\mathrm{pH}$ and DO; 2) have the capability for automated medium, feed and base additions; 3) have reduced operational requirement compared to standard STR; 4) utilize disposable vessels, thus minimizing the cleaning and steril izing procedure for a quick turn-around time; and 5) relatively high number of available vessels to be run and managed simultaneously which provide needed statistical significance for process parameters under. In this manuscript, we present the results of the study evaluating and implementing the new disposable MBR called AMBR system manufactured by The Automated Partnership (TAP Biosystems, UK) in a head-to-head comparison with 3-L bench-top STR. For this study, we compared two established MAb production processes (MAb1 \& MAb2). The study assessed the comparability of process parameters such as $\mathrm{pH}$, temperature, dissolved oxygen (DO) and osmolality between the AMBR and 3-L STR and their overall impact on cell culture performance, metabolic profiles and product quality. Furthermore, the AMBR system was utilized in a central composite DoE (Design of Experiment) based $\mathrm{pH} \&$ DO optimizations study for a MAb (MAb3) cell culture production process, and the cell culture performance results from the optimized process was confirmed and reproduced in $3-\mathrm{L}$ STR.

\section{MATERIALS AND METHODS}

\subsection{Media, Cell Lines and Culture Propagation}

Animal component-free growth medium was purchased from SAFC (St. Louis, MO). All feed media were developed in-house containing no animal-derived raw material and manufactured by SAFC and Hyclone (Logan, UT). Suspension adapted Chinese Hamster Ovary (CHO) cells lines expressing 3 different monoclonal antibodies MAb1, MAb2 and MAb3 were used. These MAb production cell lines were serially sub-cultured in growth medium. For MAb1 and MAb2 cell lines the cells were maintained in shake flasks in a $37^{\circ} \mathrm{C}$, humidified $7.5 \%$ $\mathrm{CO}_{2}$ incubator with agitation at $100 \mathrm{rpm}$. The typical cell inoculation density was $3.0-5.0 \times 10^{5}$ cells $/ \mathrm{mL}$ and the culture was routinely passaged three times per week. For MAb3 cell line the cultures were maintained and propagated as described in [9].

\subsection{Bioreactor Systems}

AMBR-24 is an automated MBR system with 24 disposable microbioreactor vessels. The AMBR instrument is housed in a bio-safety cabinet to facilitate sterile environment for sampling and feeding. The system includes two culture stations CS-1 and CS-2 containing $12 \mathrm{mi}-$ croreactors each. Each microbioreactor vessel has a miniature internal impeller, as well as individual closed loop control of dissolved oxygen, $\mathrm{pH}$ and gas supply. Each culture station has independent stirring speed and temperature control, allowing up to two different temperature and stir speeds simultaneously. AMBR system can be programmed for automated culture preparation and inoculation, feed and base addition, and culture sampling scheduling necessary for cell count, metabolite and titer analysis. Monitoring of $\mathrm{pH}$ and $\mathrm{DO}$ is via optical sensors embedded in disposable microbioreactor vessels. The measurements are performed at a frequency of every 90 seconds by the sensors.

The 3-L STR (B. Braun Biotech; Bethlehem, PA, Sartorius) were operated with a $2-\mathrm{L}$ working volume. Temperature was controlled using an electric heating blanket at $37^{\circ} \mathrm{C}$. The $\mathrm{pH}$ was maintained using a cascade of $\mathrm{CO}_{2}$ and sodium hydroxide additions. Dissolved oxygen (DO), measured as percent air saturation at $1 \mathrm{~atm}$ pressure, was maintained at set point by intermittent oxygen sparging, constant air flow in the headspace, and constant agitation. The cultures were mixed using dual pitched-blade impellers.

\subsection{MAb1 and MAb2 Cell Culture Process}

Cell inoculum scale-up was prepared in shake flasks at seeding density of $3.0-5.0 \times 10^{5}$ cells $/ \mathrm{mL}$ for both the $\mathrm{MAb}$ cultures and ready for inoculation into production vessels 2 - 3 days after initial seed. Fed batch studies in 3-L STR (2L working volume) and in AMBR (12 mL working volume) were seeded with cells at $\sim 3.0 \times 10^{5}$ cells $/ \mathrm{mL}$ obtained with a split ratio of $1: 4$. Samples were taken every 2 - 3 days to monitor for cell growth, metabolites, and MAb production. Glucose and glutamine were supplemented as needed to avoid depletion.

For initial comparison for MAb1 and MAb2, the evaluation in AMBR system was performed in replicates of 12 for each MAb, with 6 disposable microbioreactor vessels in each culture station. The positions of the MAb cell lines are depicted in (Figure 1).

Cell culture temperature was maintained at $37^{\circ} \mathrm{C}$ for the first three days and then shifted to $34^{\circ} \mathrm{C}$ for the remaining of the run. Unless indicated, media $\mathrm{pH}$ was set 
Culture Station 1

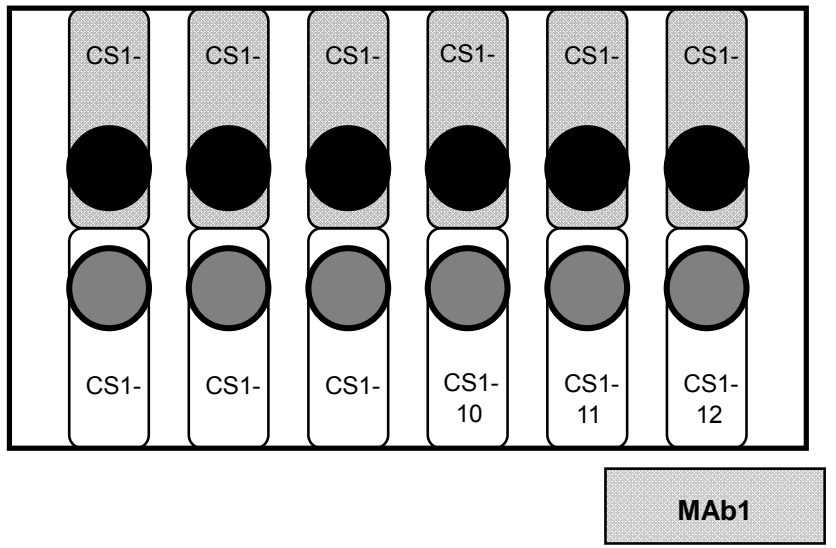

Culture Station 2

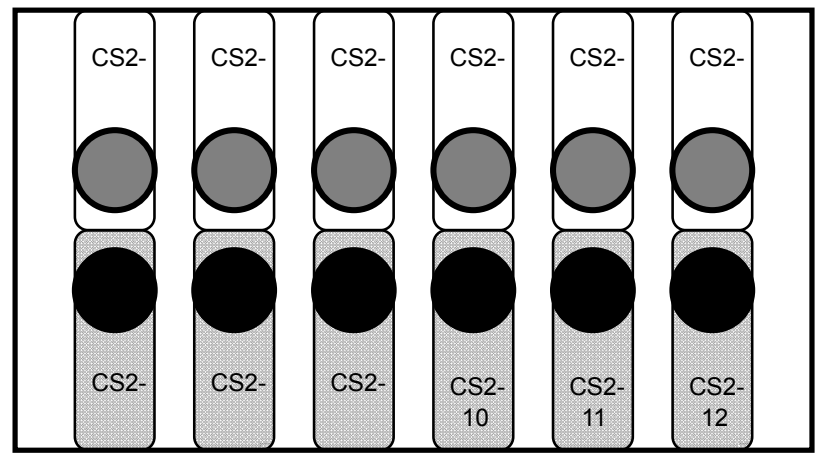

MAb2

Figure 1. Schematic representation of AMBR culture station and the experimental design set up of MAb1 and MAb2 cell line process. Each process was run in replicates of 6 in each of the culture stations.

at 6.8 with $\mathrm{pH}$ deadband at $0.05, \mathrm{DO}$ at $60 \%$ for both $3-\mathrm{L}$ and AMBR bioreactor systems, and agitation rate was set at $200 \mathrm{rpm}$ for 3-L STR and $900 \mathrm{rpm}$ for AMBR (per manufacturer's recommendation) to maintain a tip speed of $0.50 \mathrm{~m} / \mathrm{s} . \mathrm{pH}$ of the culture was controlled by addition of $1 \mathrm{M} \mathrm{NaOH}$ or sparging $\mathrm{CO}_{2}$ gas. Antifoam was added as needed during the run. For the 3-L STR, DO level was maintained at the set point with air overlay or oxygen delivered from the sparger.

\subsection{MAb3 Cell Culture Process}

Cell inoculum scale-up was prepared in shake flasks at seeding density of $2.0-4.0 \times 10^{5}$ cells $/ \mathrm{mL}$ and ready for inocula- tion into production vessels 3 - 4 days after initial seed. Fed batch studies in 3-L STR and in AMBR were seeded with cells at $\sim 2.0 \times 10^{5}$ cells $/ \mathrm{mL}$. Culture temperature was maintained at $37^{\circ} \mathrm{C}$ throughout the process. Media $\mathrm{pH}$ was set at 6.9 with $\mathrm{pH}$ deadband at 0.05 , DO at $30 \%$ for both bioreactor systems. Agitation rate was set at $200 \mathrm{rpm}$ for 3-L STR and $900 \mathrm{rpm}$ for AMBR. Samples were taken every 2 - 3 days to monitor for cell growth, metabolites, and MAb production. Glucose was supplemented as needed to avoid depletion.

\subsection{Cell Culture Analyses}

Cumulative viable cell mass, $\mathrm{CVC}$, was calculated by the following equation:

$$
\operatorname{CVC}(t)=\int_{0}^{t} X_{v}(x) \mathrm{d} x=\sum_{i=1}^{N}\left(t_{i}-t_{i-1}\right)\left(X_{v, i}+X_{v, i-1}\right) / 2
$$

Where, $X_{v}(x)$ is the viable cell density at time $x . X_{v, i}$ is the viable cell density at time $t_{i} ; X_{v, i-1}$ is the viable cell density at time $t_{i-1}$. CVC $(t)$ is the cumulative viable cell density at time $t$. The specific production rates of product were calculated as the linear regression line of the product concentration versus CVC. Statistical analyses were performed using SAS-JMP software 8.0 (SAS North Carolina, USA).

Specific glucose consumption, lactate production and lactate consumption rates were estimated by plotting cumulative glucose or lactate versus the CVC and fitting the plots with a regression coefficient of close to one. The slope of this line was used as an average specific rate

\subsection{Analytical Methods for Cell Culture and Product Quality Analysis}

Viable cell density (VCD) and total cell density in shake flasks and bioreactors were measured by CEDEX (Roche USA, Indianapolis). Glucose, lactate, glutamine, and glutamate were determined by YSI 2000 (YSI, Yellow Spring, $\mathrm{OH})$. Osmolality was detected by Advanced Micro-Osmometer (Advanced Instruments, Norwood, $\mathrm{MA}$ ). $\mathrm{pH}, \mathrm{pCO}_{2}$, $\mathrm{pO}_{2}$ were measured by ABL5 (Radiometer America Inc, Westlake, $\mathrm{OH})$.

$\mathrm{MAb}$ production in bioreactors was determined by Reversed Phase HPLC (RP-HPLC, Poros R2/10, Applied Biosystems). CVC and Qp were calculated using cell density values determined by CEDEX and MAb titers by RP-HPLC [18]. Product quality of Protein-A purified intermediates was analyzed by high performance size exclusion chromatography (HP-SEC, GE Healthcare Superdex 200 10/300 GL column), ion-exchange chromatography (IEX, Dionex Propac WCX-10 $4 \times 250 \mathrm{~mm}$ column) and reversed-phase HPLC (RP-HPLC, Poros R2/10, Applied Biosystems).

\section{RESULTS}

\subsection{Assessment of Performance between AMBR and STR}

\subsubsection{Process Parameters}

To ensure that this MBR system can mimic $\mathrm{DO}, \mathrm{pH}$, 
temperature controls observed for the 3-L STR, online profiles were collected and showed comparability be- tween the 2 bioreactor systems (Figure 2). Dissolved oxygen level of MAb1 and MAb2 process was measured

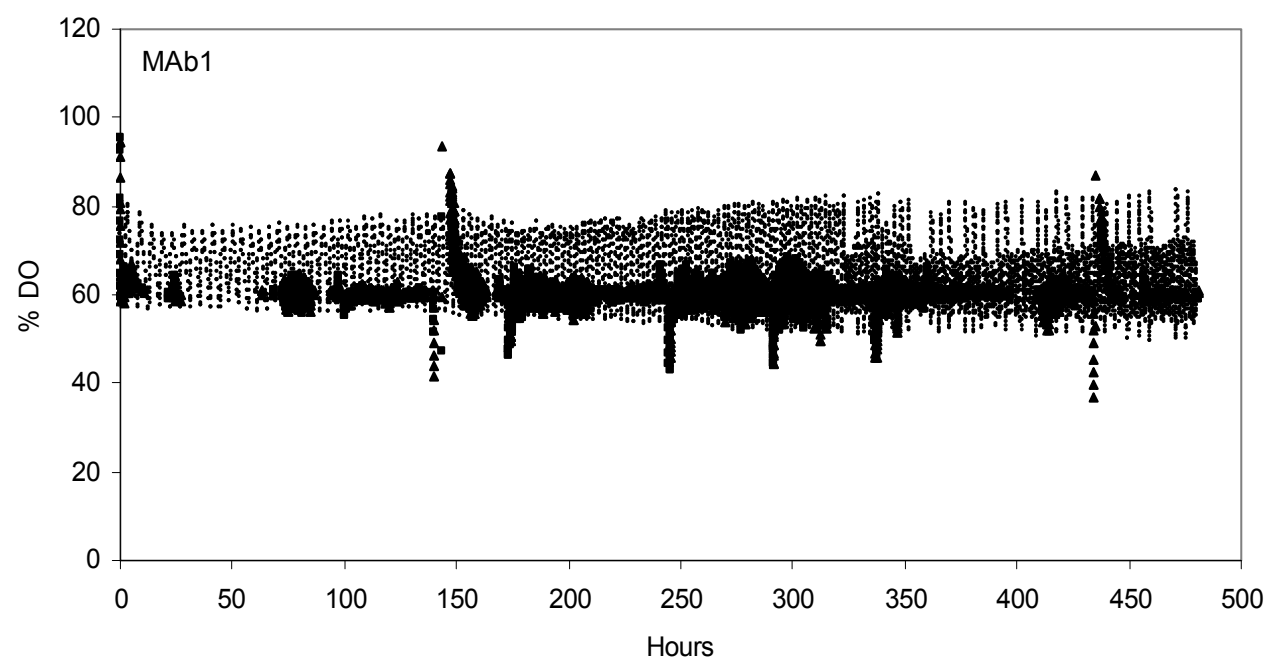

(a)

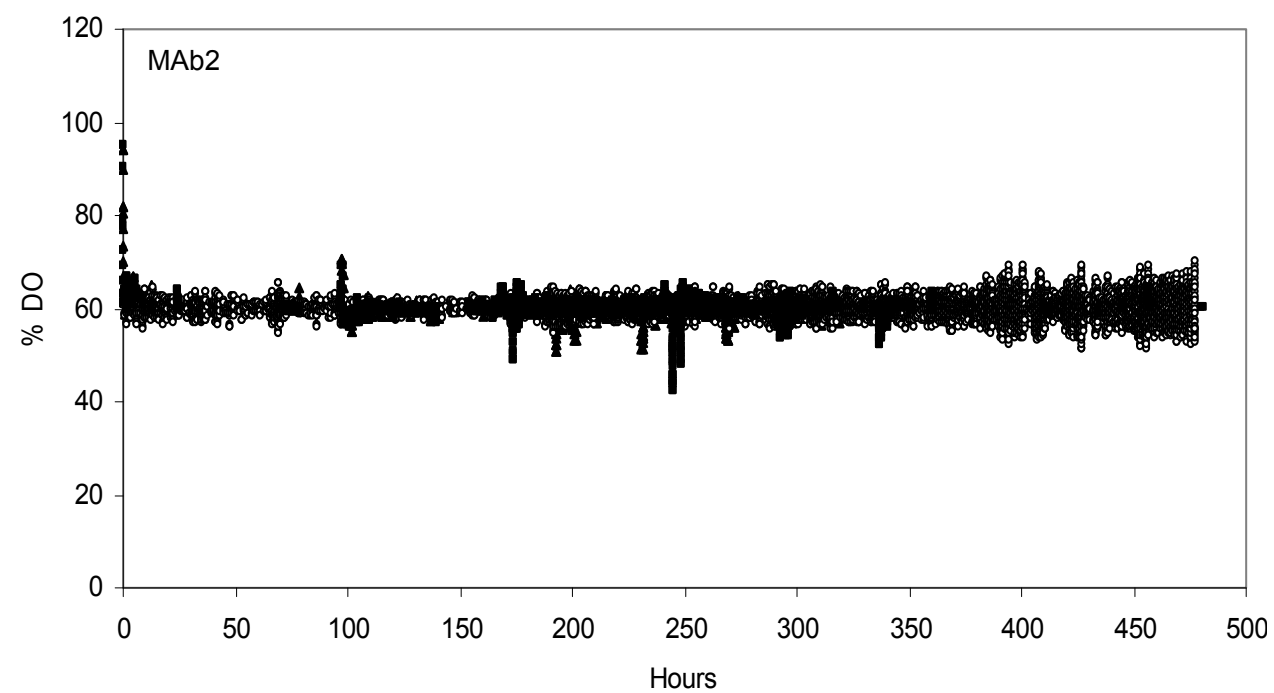

(b)

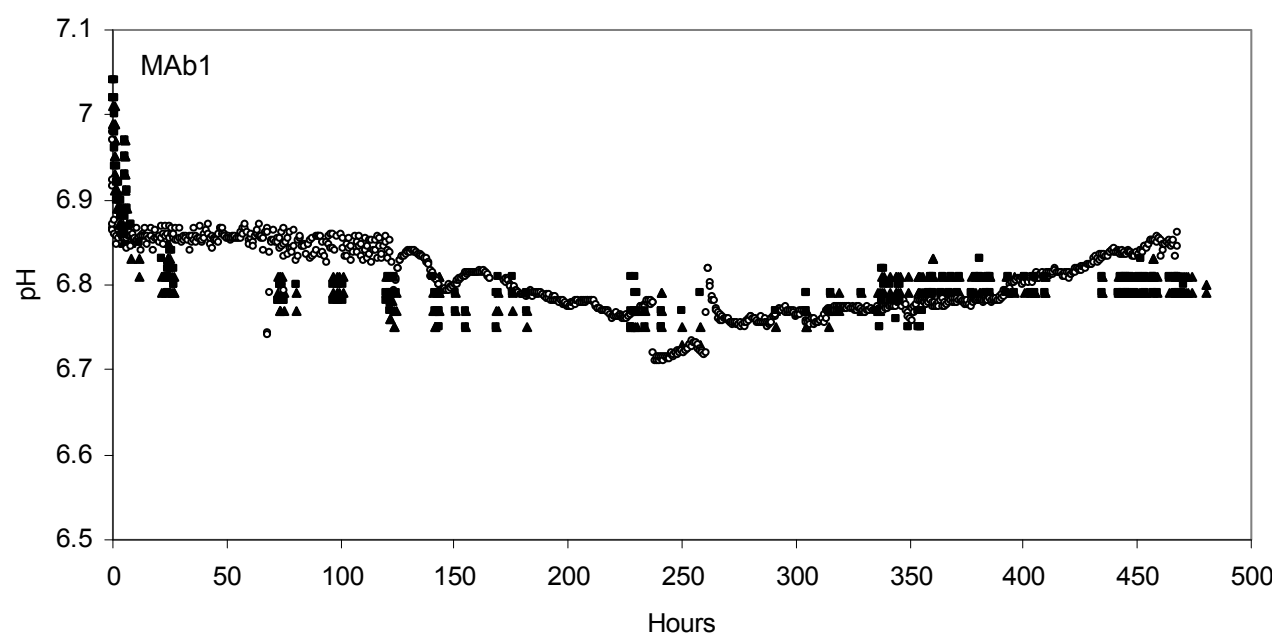

(c) 


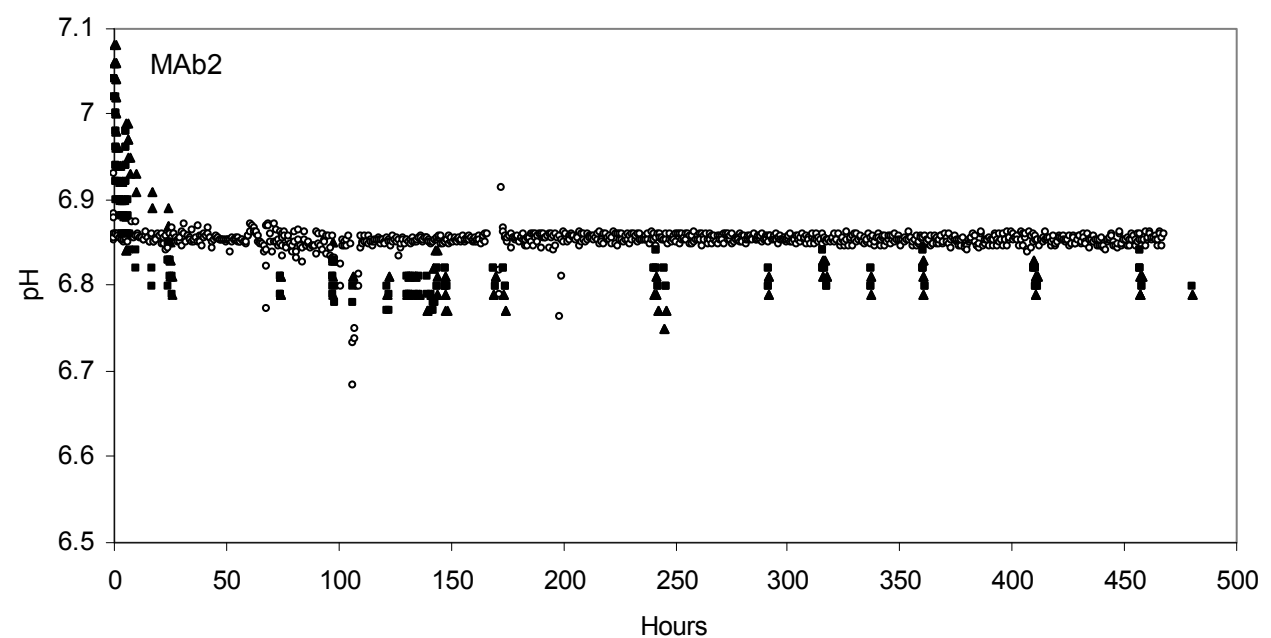

(d)

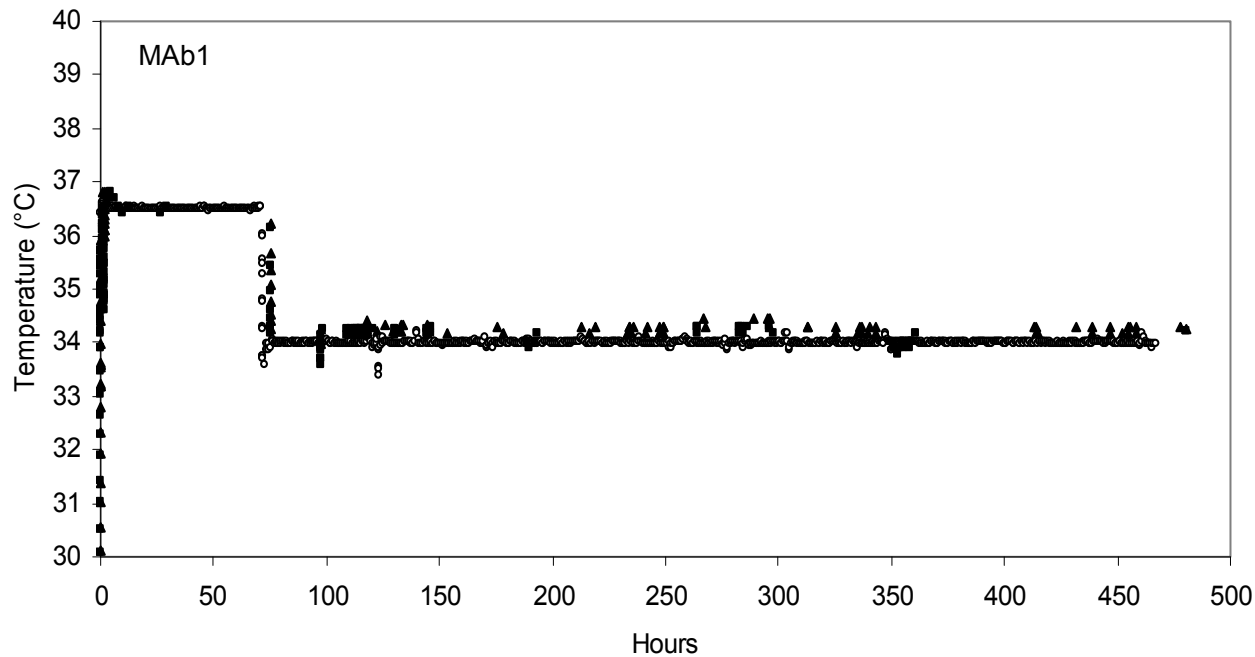

(e)

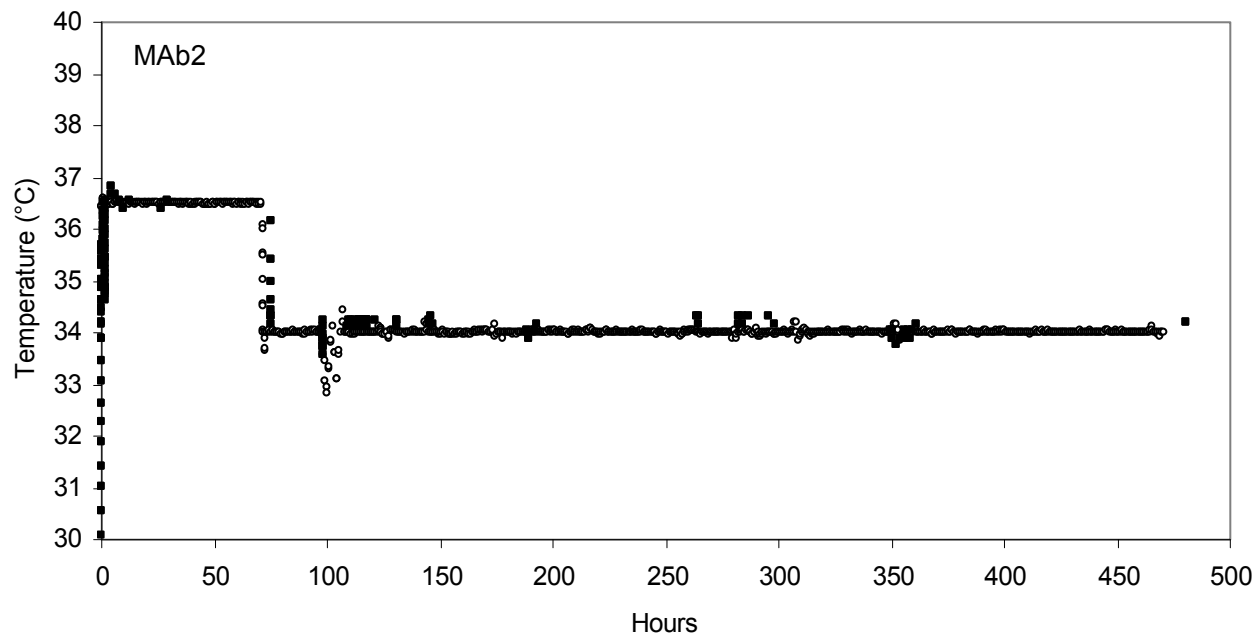

(f)

Figure 2. Online process parameter profile in AMBR and STR for both MAb1 (a), (c), (e) and MAb2 (b), (d), (f) cell lines. DO (a), (b); pH (c), (d); and temperature (e), (f). ( $\mathbf{\Delta}$ )AMBR CS-1; (a)AMBR CS-2; and (o) STR. The mean of all online values $(n=6)$ of the culture vessels from the corresponding culture stations are plotted alongside the value of 3-L STR $(n=1)$. 
online for both the AMBR and 3-L STR over the period of the process run. In MAb1 cell line the set point DO of $60 \%$ was maintained by AMBR vessels as detected by the vessel biosensors in both culture stations; however in 3-L STR the trend was maintained between $50 \%$ and $80 \%$ as detected by the DO probe. In MAb2 cell line the DO was maintained at the $60 \%$ set point in both AMBR culture stations and 3-L STR vessel.

$\mathrm{pH}$ values of MAb1 and MAb2 process was measured over the duration of the cell culture runs online in AMBR and 3-L STR (Figures 2(c) and (d)). The $\mathrm{pH}$ set point was at $\mathrm{pH} 6.8$ and dead band at 0.05 . Online values showed an acceptable variation range of \pm 0.05 from the set point between AMBR and 3-L STR and also between individual reactor vessels in each of the culture stations [17]. One of the disadvantages of earlier version of $\mathrm{pH}$ optical sensors utilized in other MBR was the loss of sensitivity to $\mathrm{pH}$ change with time, leading to the inability of these vessels to control $\mathrm{pH}$ later in culture $[3,15]$. In these demonstrated runs using the AMBR vessels embedded with optical $\mathrm{pH}$ sensors, no significant variations in $\mathrm{pH}$ readings were identified in all 24 culture vessels of the AMBR system over the 19 day of culture duration.

Each of the AMBR culture station blocks CS-1 and CS-2 can be maintained independently at different temperatures. Temperature control in AMBR is influenced by the environmental temperature [17] and care should be taken that it is maintained in an ambient condition between $21^{\circ} \mathrm{C}-25^{\circ} \mathrm{C}$ as per manufacturer's instruction. In this study AMBR measurement of culture temperature online demonstrated temperature control to be consistent with that of the 3-L STR as shown for both MAb1 and MAb2 processes (Figures 2(e) and (f)). Temperature control between the culture stations 1 and 2, as well as amongst each of the culture vessels within the stations was consistently maintained. Temperature shift was performed from $37^{\circ} \mathrm{C}$ to $34^{\circ} \mathrm{C}$ on day 3 in both the systems and maintained till the end of the run. One of the primary advantages of the AMBR over other known MBR systems is that the AMBR does not require agitation to pause for sampling or feed addition hence reducing interruption in temperature maintenance [14]. The mixing time of the feeds are also not impacted since the agitation is not affected. This characteristic feature of temperature stability in AMBR helps maintain the overall cell culture process reproducibility which can be replicated in large scale cell culture where culture temperature is not typically impacted by feed additions.

The offline trend in osmolality between the MAb1 and MAb2 process in both the AMBR and STR were shown to be similar. For MAb1 process the osmolality gradually increased from approximately 300 to 350 from day 0 to day 7 and subsequently return to 300 by the end of the culture run (Figure 3). For MAb2 process the offline osmolality peaked on day 6 to approximately 375 and decreased to 350 by the end of the culture run. The comparable process parameter results detected in 2 individual cultures with independent processes from the AMBR and the 3-L STR demonstrate the AMBR has the potential to replicate the performance of bench-scale bioreactors.

\subsubsection{Cell Culture Parameters}

VCD and viability of both MAb1 and MAb2 cell lines were analyzed offline using CEDEX at regular intervals during the process and showed consistency between the AMBR stations and 3-L STR throughout the culture runs (Figure 4(a)). A peak cell density of approximately 4.0 $5.0 \times 10^{6}$ cells $/ \mathrm{mL}$ and $6.0-7.0 \times 10^{6}$ cells $/ \mathrm{mL}$ was achieved in both AMBR and 3-L STR on day 10 for MAb1 and MAb2 cell lines respectively. Viability was maintained at $\sim 95 \%$ until day 12 for both MAb1 and MAb2 in AMBR and 3-L STR after which it trended down gradually to $80 \%$ for MAb1 and $60 \%$ for MAb2 cell lines at the time of harvest (Figure 4(b)). The plot also shows overall consistency in VCD and viability between culture stations in AMBR in each of the processes.

Protein production in both MAb1 and MAb2 cell culture process followed a consistent trend between AMBR and 3-L STRs. The final titer at the end of the production batches were approximately $1.2 \pm 0.2$ and $1.0 \pm 0.1$ arbitrary units for MAb1 and MAb2 respectively in both AMBR and 3-L STRs (Figure 4(c)). More importantly, protein quality attributes of Protein-A purified MAb from the AMBR and STR systems determined by RP-HPLC, HP-SEC (Figure 4(d)) and IEX (Figure 4(e)) indicated comparable product quality profiles.

The metabolic profile for both MAb1 and MAb2 cell lines were measured and represented as rates (Table 1).

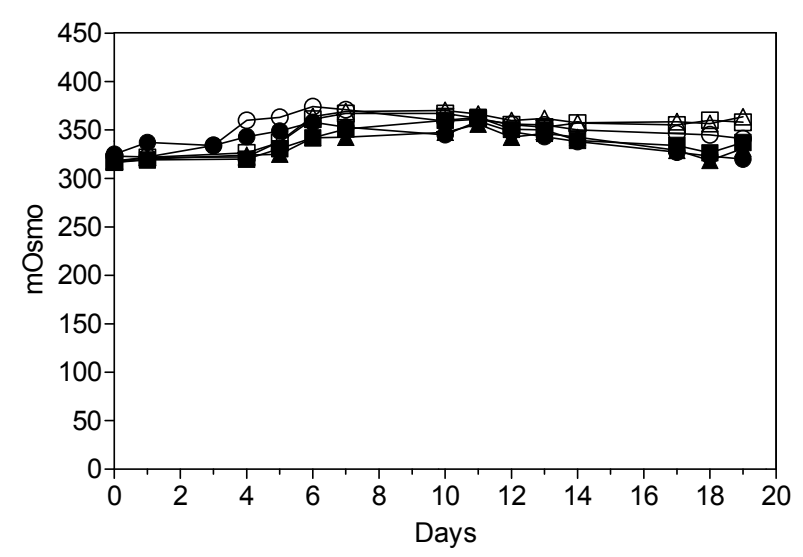

Figure 3. Offline measurement of Osmolality for AMBR and STR in both MAb1 and MAb2 processes. ( $\boldsymbol{\Delta})$ MAb1:AMBR

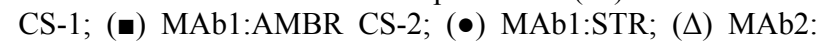
AMBR CS-1; ( $\square$ ) MAb2:AMBR CS-2; and (०) MAb2: STR. The mean of all online values $(n=6)$ of the culture vessels from the corresponding culture stations are plotted alongside the value of $3-\operatorname{L~STR~}(n=1)$. 


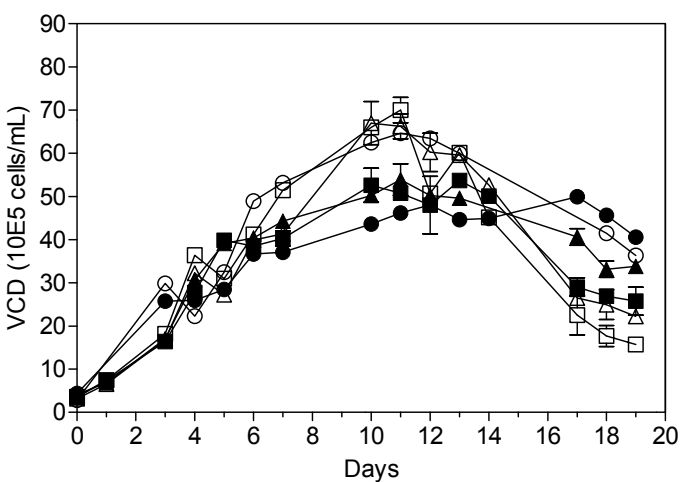

(a)

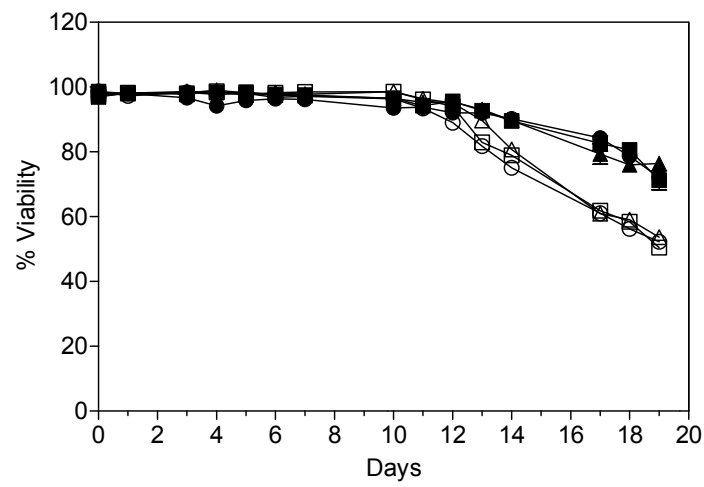

(b)

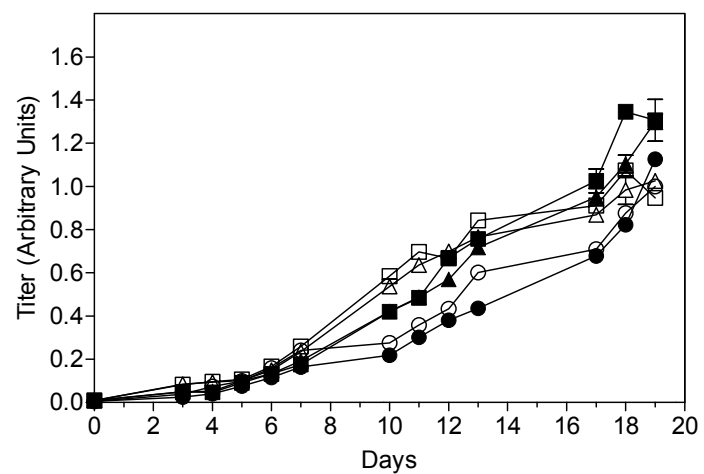

(c)

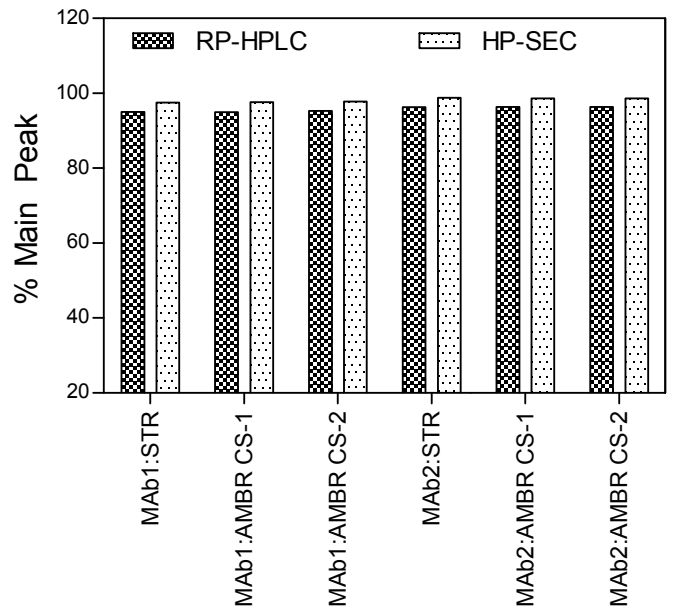

(d)

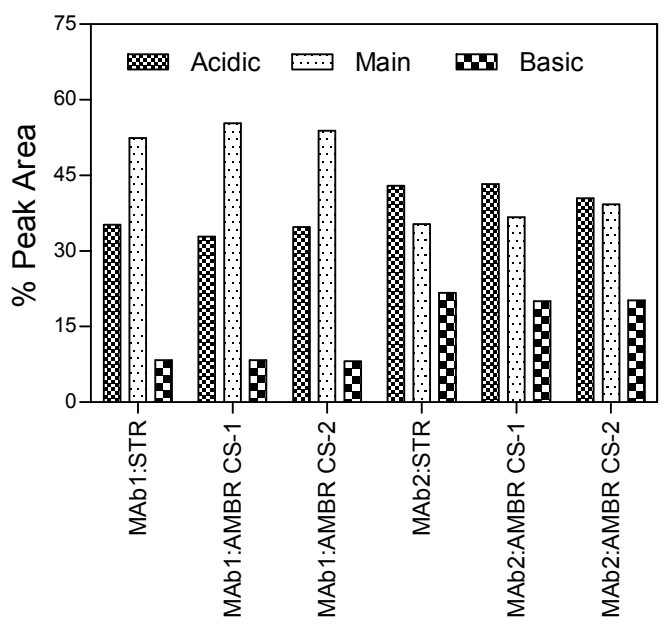

(e)

Figure 4. Offline monitoring of (a) viable cell density, (b) cell viability, (c) MAb titer. ( $\mathbf{\Delta})$ MAb1: AMBR CS-1; (ロ) MAb1:AMBR CS-2; (•) MAb1: STR; ( $\triangle$ ) MAb2:AMBR CS-1; ( $\square)$ MAb2:AMBR CS-2; and (O) MAb2: STR. Product quality assessment for MAb1 and MAb2 cell culture processes in AMBR and STR by (d) RP-HPLC and HP-SEC (e) IEX. The mean of all online values $(\mathrm{n}=$ 6) of the culture vessels from the corresponding culture stations are plotted alongside the value of 3-L STR $(\mathrm{n}=1)$.

Table 1. Metabolic profiles for MAb1 and MAb2 processes between AMBR and STR.

\begin{tabular}{|c|c|c|c|}
\hline Bioreactor & $\begin{array}{c}\text { Glucose } \\
\text { Consumption } \\
\text { Rate }^{*}\end{array}$ & $\begin{array}{l}\text { Lactate } \\
\text { Production } \\
\text { Rate }^{* 1}\end{array}$ & $\begin{array}{c}\text { Lactate } \\
\text { Consumption } \\
\text { Rate }^{* 2}\end{array}$ \\
\hline & & MAb1 Process & \\
\hline AMBR CS $-1^{\S}$ & $-428.8 \pm 6.0$ & $218.2 \pm 5.7$ & $-21.8 \pm 1.5$ \\
\hline AMBR CS- $2^{\S}$ & $-404.1 \pm 4.8$ & $221.1 \pm 6.0$ & $-21.1 \pm 1.1$ \\
\hline \multirow[t]{2}{*}{ 3-L STR ${ }^{\phi}$} & -447.1 & 162.4 & -18 \\
\hline & & MAb2 Process & \\
\hline AMBR CS- 1 \& & $-356.4 \pm 4.4$ & $242.3 \pm 14.7$ & $-14.6 \pm 2.4$ \\
\hline AMBR CS- $2^{\S}$ & $-347.9 \pm 12.3$ & $245.6 \pm 36.2$ & $-14.2 \pm 0.7$ \\
\hline 3-L STR ${ }^{\phi}$ & -478.0 & 220.4 & -11.6 \\
\hline
\end{tabular}

${ }^{*}$ All rates are represented as pg/cell-day; ${ }^{\S} \mathrm{n}=6 ;{ }_{\mathrm{n}}=1 ;{ }^{1}$ Lactate production during early production phase; ${ }^{2}$ Lactate consumption during later production phase.

The initial consumption rates of glucose for MAb1 process were similar across the different culture stations of the AMBR and 3-L STR. However the glucose utilizetion rate was higher for the MAb2 process in 3-L STR at $\sim 478 \mathrm{pg} /$ cell-day compared to those from the AMBR culture stations with an average rate of $\sim 350 \mathrm{pg} /$ cell-day. The lactate production rates for MAb1 process in $3-\mathrm{L}$ STRs ( $162 \mathrm{pg} /$ cell-day) showed a decrease of $\sim 26 \%$ compared to the two culture stations in AMBR (average 
$\sim 219 \mathrm{pg} /$ cell-day). Conversely the lactate production rates for MAb2 process were similar between the AMBR and 3-L STRs at $\sim 244 \mathrm{pg} /$ cell-day and $\sim 220 \mathrm{pg} /$ cell-day, respectively. The lactate consumption rates during the later stage of the cell culture runs followed similar trending of lower consumption rates resulted from lower production rates for both MAb1 and MAb2 processes.

\subsection{Optimizing the Effect of $\mathrm{pH}$ and $\mathrm{DO}$ of an MAb Process Using AMBR}

The comparable online profiles of $\mathrm{pH}$ and DO demonstrated for MAb1 and MAb2 between the STR and the AMBR provided confidence that initial $\mathrm{pH}$ and $\mathrm{DO}$ process optimization can be performed in a high throughput manner using the AMBR. For MAb3, an initial $\mathrm{pH}$ and DO screening was conducted with the AMBR to enhance MAb3 productivity (Figure 5). Multivariate analysis (Figure 5(a)) for $\mathrm{pH}, \mathrm{DO}$ and titer demonstrated that $\mathrm{pH}$ set point had a significant effect on MAb titer $(\mathrm{p}<$ $0.05)$ while DO did not $(p>0.05)$. Regression analyses of specific productivity $(\mathrm{Qp})$ as a function of $\mathrm{pH}$ setpoints demonstrated positive linear relationship (Figure 5(b)). The increase in titer productivity could also be attributed to the increase in the $\mathrm{CVC}$ at higher $\mathrm{pH}$ ranges (Figure 5(c)). Overall $\mathrm{pH} \mathrm{7.0,} \mathrm{the} \mathrm{highest} \mathrm{pH}$ setpoint tested and DO at $30 \%$ were determined to be the optimal process condition for further confirmation in 3-L STR.

To assess the scalability of the AMBR optimized cell culture process, 4 replicate runs were conducted in $3-\mathrm{L}$ STR with chosen setpoints of $\mathrm{pH}$ at 7.0 and $\mathrm{DO}$ at $30 \%$. As shown in (Figures 5(d) and (e)), the cell culture performance in 3-L STR as represented by CVC and titer productivity of MAb3 cell line process was found to be comparable and more importantly scalable with the data obtained from AMBR.

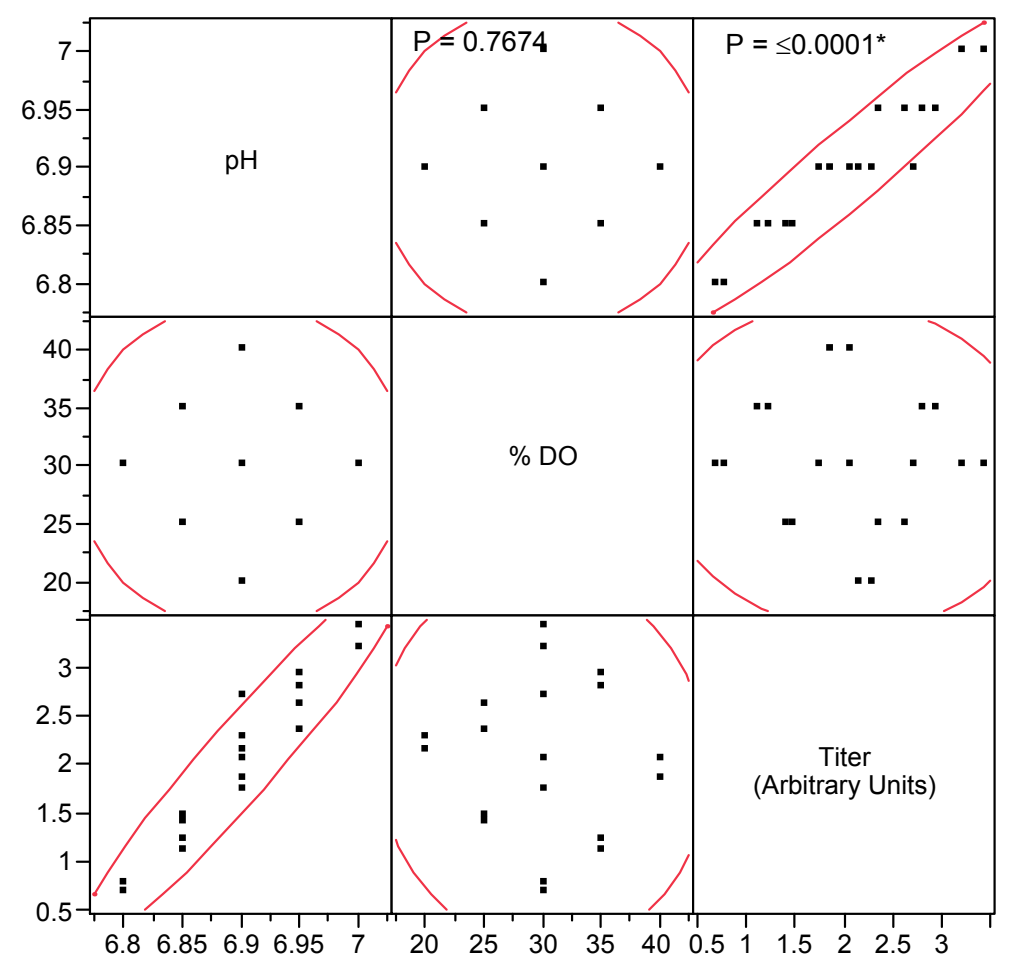

(a)

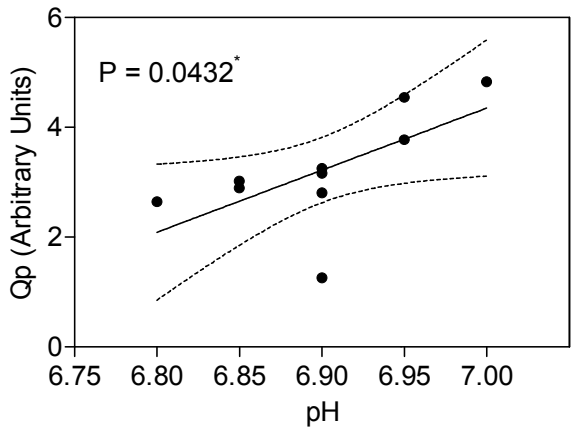

(b)

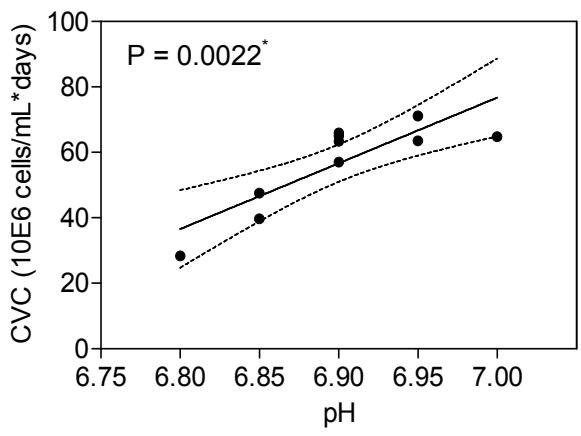

(c) 


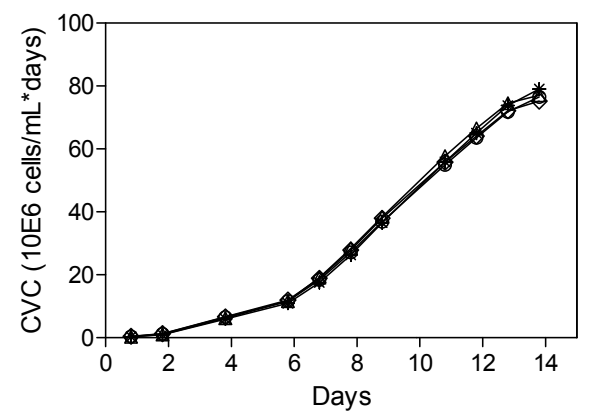

(d)

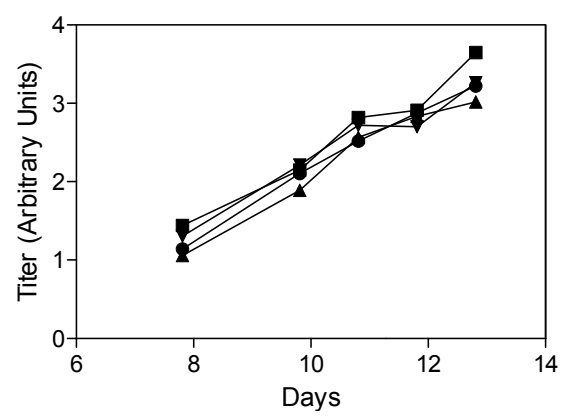

(e)

Figure 5. Optimizing $\mathrm{pH}$ and $\mathrm{DO}$ set points of MAb3 process in AMBR and statistical analyses of test conditions. (a) titer, (b) Qp, (c) CVC. Evaluation of AMBR optimized pH and DO in 3-L STR as quadruplicates (d) CVC and (e) titer.

\section{DISCUSSION \& CONCLUSION}

Cell culture process development timeline is driven by historical experience and existence of platform. However, new MAb molecules entering the development pipeline are required to not only meet specific product quality profiles, but also develop in shorten timelines. As a result, high throughput screens to develop production processes become critical path to meet these demands. The emergence of single use disposable bench-scale and largescale bioreactors has facilitated rapid development and manufacturing of drug supplies. We demonstrate a similar approach is applicable for high throughput process development. While disposable bench-scale bioreactors can reduce set-up and break-down time, no time or resource saving was apparent in cell inoculum and medium preparation or batch operation. We have selected the use of AMBR system fitted with disposable microbioreactors for process development, as they have low inoculum and medium requirement and thus minimize operational workload and improve throughput. The system also has a well integrated technology for automated inoculation, feeding and sampling. The disposable nature of the AMBR vessels reduces vessel set-up and clean-up effort.

Based on our studies, the AMBR system is shown to be a versatile platform for HTP process development and optimization for mammalian cell cultures. We have demonstrated that the AMBR is an excellent and efficient tool to support a number of HTP DoE-based process development investigations. These include identification of feed medium concentrations, $\mathrm{pH}$ and/or DO optimization ranges for a mammalian cell line, comparison of culture performance of multiple cell lines with the similar processes, or conducting process ranging studies. The utilizetion of HTP DoE-based investigation is most advanta geous during initial stage of process development as this system enhances data outputs with reduced timeline, and thus allows program decision for cell culture area to proceed earlier. Nevertheless, early process ranging studies conducted using this HTP DoE-based approach to narrow the process test ranges would significant reduce the more labor intensive, subsequent work in small scale STR.

While our results are supportive of the AMBR in meeting most of the STR capabilities, there are several points which would allow AMBR or other MBR systems to achieve more desirable operational controls and the potential to be a scale-down model for large-scale vessels. First, continuous feed strategy cannot be achieved. An attempt to design a continuous feed strategy with the MBR will require multiple and frequent small feed aliquot additions. Since this MBR system is not a closed system, introduction of frequent feed additions would change the gassing regime and may affect the overall $\mathrm{pH}$ and DO of some cell cultures. Second, continuous sparging in the MBR necessitates frequent antifoam additions that may not be required for some cultures in standard STR. Third, culture temperature control in the MBRs like AMBR can be affected by environmental temperature. Fourth, the current state of this MBR vessel and impeller configurations are different from those of the standard STR, and thus processes developed and $\mathrm{K}_{\mathrm{La}}$ calculated using this MBR may not be scalable to larger vessels for some cultures. With further development and design modification these limitations could be addressed in the future.

\section{REFERENCES}

[1] ICH Guidelines (2011) ICH quality implementation working group points to consider (R2) I CH-Endorsed Guide for ICH Q8/Q9/Q10/Q11 Implementation. 6 December 2011.

[2] Kumar, S., Wittmann, C. and Heinzle, E. (2004) Minibioreactors. Biotechnology Letters, 26, 1-10.

[3] Betts, J.I. and Baganz, F. (2006) Miniature bioreactors: Current practices and future opportunities. Microbial Cell Factories, 5, 21. doi:10.1186/1475-2859-5-21

[4] Schapper, D., Alam, M.N., Szita, N., Eliasson Lantz, A. and Gernaey, K.V. (2009) Application of microbioreac- 
tors in fermentation process development: A review. Analytical and Bioanaytical Chemistry, 395, 679-695.

[5] Bareither, R. and Pollard, D.A. (2010) A review of advanced small-scale parallel bioreactor technology for accelerated process development: Current state and future need. Biotechnology Progress, 27, 2-14.

[6] Girard, P., Jordan, M., Tsao, M. and Wurm, F.M. (2001) Small-scale bioreactor system for process development and optimization. Biochemical Engineering Journal, 7, 117-119.

[7] John, G.T., Klimant, I., Wittmann, C. and Heinzle, E. (2003) Integrated optical sensing of dissolved oxygen in microtiter plates: A novel tool for microbial cultivation. Biotechnology Bioengineering, 81, 829-836. doi:10.1002/bit.10534

[8] Szita, N., Boccazzi, P., Zhang, Z., Boyle, P., Sinskey, A.J. and Jensen, K.F. (2005) Development of a multiplexed microbioreactor system for high-throughput bioprocessing. Lab Chip, 5, 819-826. doi:10.1039/b504243g

[9] Amanullah, A., Otero, J.M., Mikola, M., Hsu, A., Zhang, J., Aunins, J., Schreyer, B.H., Hope, J.A. and Russo, P.A. (2010) Novel micro-bioreactor high throughput technology for cell culture process development: Reproducibility and scalability assessment of fed-batch $\mathrm{CHO}$ cultures. Biotechnology Bioengineering, 106, 57-67.

[10] Micheletti, M. and Lye, G.J. (2006) Microscale bioprocess optimisation. Current Opinion in Biotechnology, 17, 611-618.

[11] Mazzei, D., Guzzardi, M.A., Giusti, S. and Ahluwalia, A. (2010) A low shear stress modular bioreactor for connected cell culture under high flow rates. Biotechnology
Bioengineering, 106, 127-137.

[12] Wen, Y., Zang, R., Zhang, X. and Yang, S.T. (2012) A 24 microwell plate with improved mixing and scalable performance for high throughput cell cultures. Process Biochemistry, 47, 612-618. doi:10.1016/j.procbio.2011.12.023

[13] Harms, P., Kostov, Y., French, J.A., Soliman, M., Anjanappa, M., Ram, A. and Rao, G. (2006) Design and performance of a 24-station high throughput microbioreactor. Biotechnology Bioengineering, 93, 6-13. doi:10.1002/bit.20742

[14] Isett, K., George, H., Herber, W. and Amanullah, A. (2007) Twenty-four-well plate miniature bioreactor high-throughput system: Assessment for microbial cultivations. Biotechnology Bioengineering, 98, 1017-1028.

[15] Chen, A., Chitta, R., Chang, D. and Amanullah, A. (2009) Twenty-four well plate miniature bioreactor system as a scale-down model for cell culture process development. Biotechnology Bioengineering, 102, 148-160.

[16] Lewis, G., Lugg, R., Lee, K. and Wales, R. (2010) Novel automated micro-scale bioreactor technology: A qualitative and quantitative mimic for early process development. Bioprocessing Journal, 9, 22-25.

[17] Hsu, W.T., Aulakh, R.P., Traul, D.L. and Yuk, I.H. (2012) Advanced microscale bioreactor system: A representative scale-down model for bench-top bioreactors. Cytotechnology, 64, 667-678. doi:10.1007/s10616-012-9446-1

[18] Manahan, M.S. and Ling, W.L. (2009) Profiling intracellular monoclonal antibody expression for improved cell culture characterization and process optimization. Bioprocessing Journal, 8, 22-27. 